\title{
Differential regulation of diacylglycerol kinase isoform in human failing hearts
}

\author{
Olga Bilim¹, Tetsuro Shishido ${ }^{1 *}$, Shuji Toyama², Satoshi Suzuki ${ }^{3}$, Toshiki Sasaki ${ }^{1}$, Tatsuro Kitahara', \\ Mitsuaki Sadahiro ${ }^{2}$, Yasuchika Takeishi ${ }^{3}$ and Isao Kubota ${ }^{1}$
}

\begin{abstract}
Evidence from several studies indicates the importance of Gaq protein-coupled receptor (GPCR) signaling pathway, which includes diacylglycerol (DAG), and protein kinase $C$, in the development of heart failure. DAG kinase (DGK) acts as an endogenous regulator of GPCR signaling pathway by catalyzing and regulating DAG. Expressions of DGK isoforms $\alpha, \varepsilon$, and $\zeta$ in rodent hearts have been detected; however, the expression and alteration of DGK isoforms in a failing human heart has not yet been examined. In this study, we detected mRNA expressions of DGK isoforms $\gamma, \eta, \varepsilon$, and $\zeta$ in failing human heart samples obtained from patients undergoing cardiovascular surgery with cardiopulmonary bypass. Furthermore, we investigated modulation of DGK isoform expression in these hearts. We

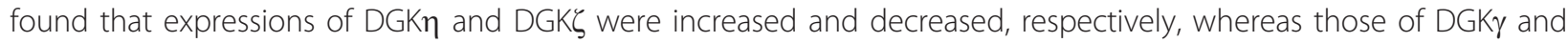
$D G K \varepsilon$ remained unchanged. This is the first report that describes the differential regulation of DGK isoforms in normal and failing human hearts.
\end{abstract}

\section{Introduction}

Epidemiological studies have suggested that cardiac hypertrophy is an independent risk factor for the development of heart failure and is associated with increased cardiac morbidity and mortality in patients with cardiovascular diseases [1-3]. Recent in vivo and in vitro studies have focused on protein kinase signaling cascades as the molecular mechanisms regulating the hypertrophic response of cardiomyocytes [4,5]. Among these signaling pathways, the Gaq protein-coupled receptor (GPCR) signaling pathway, which includes diacylglycerol (DAG) and protein kinase C (PKC), plays a critical role in the development of cardiac hypertrophy and progression to heart failure (HF) [6-8].

The main route for termination of DAG signaling is through phosphorylation by DAG kinase (DGK) to produce phosphatidic acid $[9,10]$. To date, at least 10 DGK isoforms-DGK $\alpha, \beta, \gamma, \delta, \varepsilon, \zeta, \eta, \theta$, l, and $\kappa$ - have been identified in mammals; DGK isoforms have been reported to be expressed in various tissues, suggesting the importance of these kinases in basic cellular functions $[11,12]$. In rodent hearts, the expressions of

\footnotetext{
* Correspondence: tshishid@med.id.yamagata-u.ac.jp

'Department of Cardiology, Pulmonology, and Nephrology, Yamagata

University School of Medicine, Yamagata, Japan

Full list of author information is available at the end of the article
}

DGK $\alpha, \varepsilon$, and $\zeta$ isoforms have been detected, and differential regulation of DGK isozymes in the development of pressure-overload cardiac hypertrophy and in left ventricular remodeling after myocardial infarction has been shown $[13,14]$. Evidence from several in vitro [15] and in vivo [16] studies suggests that DGK $\zeta$ blocks GPCR-induced activation of PKC, and suppresses cardiomyocyte hypertrophy and progression of heart failure.

However, the expression of DGK isoforms in failing human heart has not been previously examined. Therefore, the purpose of this study was (1) to identify the DGK isoforms in the right atrial myocardium in patients undergoing cardiac surgery with cardiopulmonary bypass and (2) to examine changes in expressions of DGK isoforms in cases of failing human heart due to chronic volume overload.

\section{Materials and methods}

\section{Study patients and materials}

Intraoperative samples of the right atrial myocardium were obtained from a total of 17 patients who underwent cardiac surgery at the Yamagata University Hospital between February 2006 and September 2007. All procedures were performed in accordance with the ethical standards outlined in the Declaration of Helsinki of 1975 (revised 1983). The research protocol was 
approved by the institution's ethical committee, and written informed consent was obtained from all subjects.

Heart samples were obtained from 10 consecutive patients [mean age: mean (SD), 63 (13) years; 7 men and 3 women] admitted for surgical correction of chronic regurgitation associated with mitral valvular lesions (valvular replacement or valvuloplasty, $\mathrm{n}=6$ ) or combined dual-valve replacement $(\mathrm{n}=4)$ (Table 1$)$. Right atrial tissue samples collected from 7 patients with aortic dissection, no structural cardiac diseases, and normal heart function were used as controls. Small samples of the right atrium tissue were collected when patients underwent median sternotomy with aortic and right atrial cannulation. The samples were obtained in the operating room and rapidly frozen in liquid nitrogen until further use.

\section{RNA preparation and reverse transcription-polymerase chain reaction analysis}

Extraction of DNA-free total cardiac RNA was performed using the RNeasy fibrous tissue mini kit (Qiagen, Tokyo, Japan) according to the manufacturer's instructions. For conventional reverse transcriptase polymerase chain reaction (RT-PCR) analysis, $1 \mu \mathrm{g}$ of total RNA was reverse-transcribed using the QuantiTect reverse transcription kit (Qiagen) $[17,18]$. The primer pairs for human DGK isoforms used for PCR analysis were designed on the basis of GenBank sequences (DGK $\alpha, \quad B C 031870 ; \quad D G K \beta, \quad A B 018261 ;$ DGK $\gamma$,

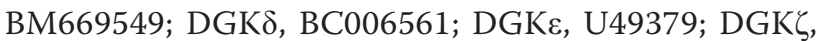

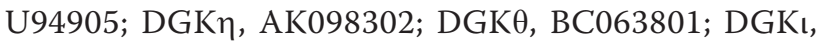
AF061936; DGK $\kappa$, AB183864; GAPDH, M33197). PCR products were characterized by performing agarose gel electrophoresis on $2 \%$ Tris/borate/EDTA (TBE) agarose gel and visualized by ethidium bromide staining. Densitometry of the bands was performed using ImageJ (v1.29s NIH). The intensities of the bands were normalized for GAPDH. Each reaction included positive and negative controls. Total RNA from Human brain (Ambion, Cat. No. AM7962) and HeLa cells were used as positive controls.

\section{Statistical Analysis}

Data are presented as mean (SD). Differences between the 2 groups were evaluated using Student's $t$ test, and a $P$-value of $<0.05$ was considered statistically significant. All statistical analyses were performed with a standard statistical program package (JMP version 8; SAS Institute Inc., Cary, North Carolina).

\section{Results}

Analysis of DGK isoform expression in a normal heart

First, we confirmed the expression of DGK $\alpha, \beta, \gamma, \varepsilon, \zeta$, $\eta, \theta$, and $\mathrm{\iota}$ in human brain cells and that of DGK $\delta, \varepsilon$, $\zeta$, and $\eta$ in the HeLa cell line by using RT-PCR (data not shown). The human brain or HeLa cells did not show the expression of DGK $\kappa$. This finding is consistent with that reported by a recent study that showed the presence of DGK $\kappa$ mRNA only in the human testis and placenta tissues [19]. Therefore, we used mRNA from the human brain and HeLa cells as positive control for further experiments using human heart tissue.

\section{Clinical and hemodynamic characteristics of patients with heart failure}

Clinical characteristics and echocardiographic and hemodynamic measurements of heart failure patients undergoing valvular replacement or valvuloplasty are shown in Table 1. Five patients showed New York Heart Association (NYHA) functional class III heart failure, and another 5 patients showed class IV heart failure. Echocardiography revealed that the patients had marked left ventricular dilatation (LVDD, 64 (7) $\mathrm{mm}$ vs. $47(4) \mathrm{mm}$ in control, $\mathrm{P}<0.0001)$ and left atrium dilatation (LAD, 56 (13) $\mathrm{mm}$ vs. 35 (6) $\mathrm{mm}$ in control, $\mathrm{P}=0.0011)$. Six patients had atrial fibrillation. Five patients had low cardiac index $\left(<2.5 \mathrm{~L} / \mathrm{min} / \mathrm{m}^{2}\right.$. Elevation of pulmonary arterial pressure (PAP systolic $\geq 30 \mathrm{mmHg}$ ), pulmonary capillary wedge pressure (mean of PCWP $\geq 12 \mathrm{mmHg}$ ), and right ventricular pressure (RVP systolic $\geq 30 \mathrm{mmHg}$ ) was observed in 7 , 6 , and 8 patients, respectively. Echocardiography revealed moderate to severe tricuspid regurgitation in 4 patients. A large proportion of patients had right atrial overload.

The expression of the DGK isoforms in the human right atrium was examined in the control heart specimens by using RT-PCR. RT-PCR analysis performed using oligonucleotide primers specific for the 10 human DGK isoforms revealed 4 DGK isoforms DGK $\gamma$,

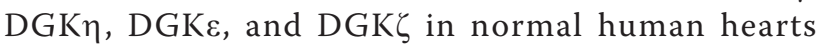
(Figure 1).

\section{Changes in DGK isoform expression in hearts with volume-overload}

To investigate the changes in mRNA levels of the DGK isoforms in patients with volume-overloaded atria, we

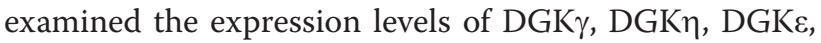
and $D G K \zeta$ isoforms in the right atrium specimens obtained from heart failure patients and compared them with the corresponding expression levels in the control heart samples. Volume overload caused changes in the expression levels of DGK $\eta$ and DGK . Expression level of DGK $\eta$ was significantly increased (Figure 2A), while that of DGK $\zeta$ was significantly decreased (Figure 2B). In contrast, expression levels of DGK $\gamma$ and DGK $\varepsilon$ remained unchanged in the patients with chronic overload in the right atrium. 


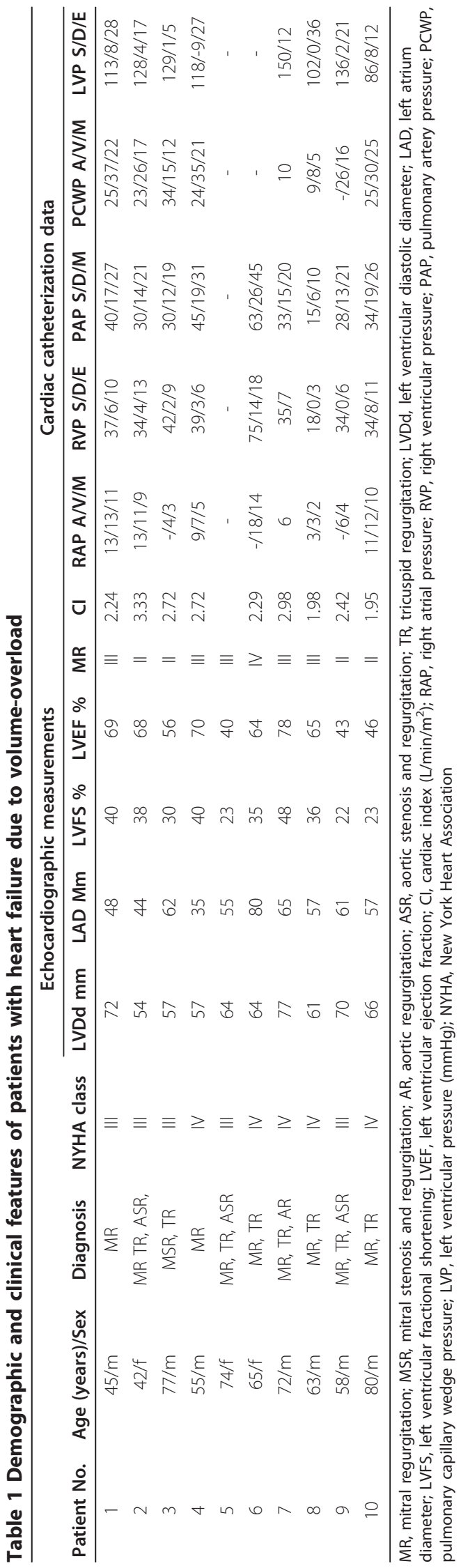




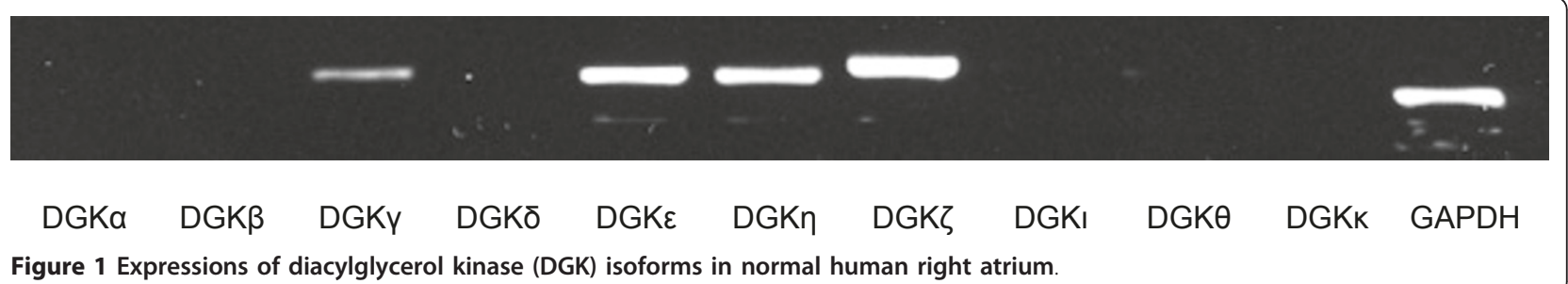

\section{Discussion}

All DGK family members share conserved domains and are subdivided into 5 functional classes on the basis of the subtype-specific regulatory domains [12]. DGK represents a large family of isoforms that differ remarkably in their structure, tissue expression, and enzymatic properties, and are encoded by different genes [11]; however, to the best of our knowledge, DGK isoform expression in the human heart has not been previously examined.

In the present study, we used the right atrium tissue to determine the expression of DGK isoforms. Chronic mitral regurgitation is a state of volume overload that causes complex hemodynamic changes [20-22]. Chronic mitral insufficiency leads to the enlargement of the left atrium, pulmonary congestion, and failure of the right heart. Pulmonary hypertension occurs frequently (in $76 \%$ of cases) in patients with isolated chronic mitral regurgitation with preserved left ventricular systolic function [23]. Samples of the left ventricular myocardium obtained from patients who were undergoing orthotopic cardiac transplantation have been used in several studies, thereby suggesting that the hearts were in the state of end-stage in most cases, and were modified by endogenous and exogenous stimuli $[24,25]$. In the light of these facts, in this study, the right atrium samples were obtained from patients with chronically stressed hearts; these samples were suitable for determining the clinical significance of DGK in modulation of progressive heart failure.

We detected 4 DGK isoforms belonging to 4 different classes in the human heart. Unlike DGKa expression in

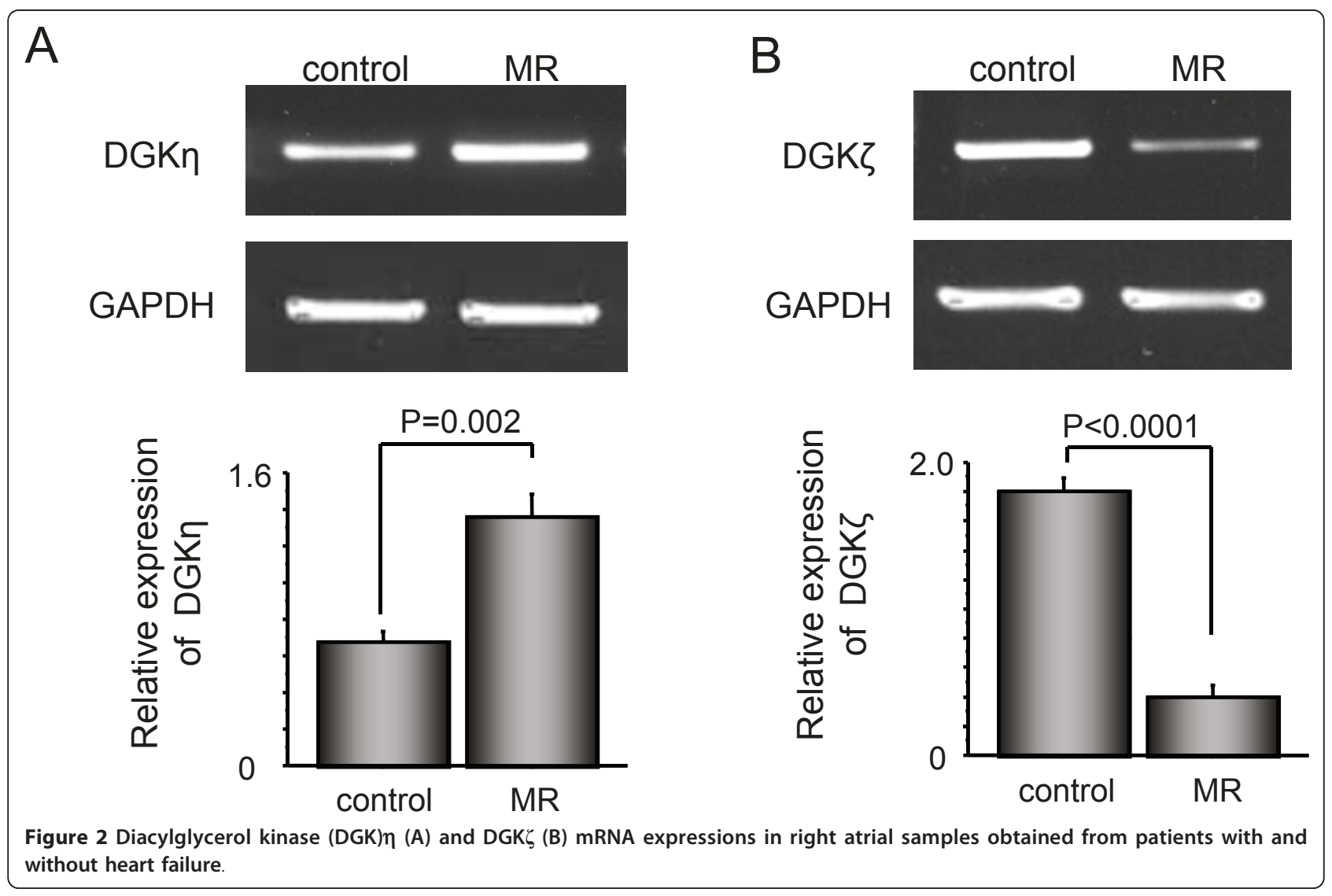


rodent hearts, DGK $\gamma$, another class I DGK, was expressed in the human heart, thereby implying that DGK $\alpha$ in the rodent model can be applied as a molecular target for confirming the clinical significance of DGK $\gamma$ in the human heart. Although no changes were detected in the expression level of DGK $\gamma$ in failing heart, we suspected that DGK $\gamma$ might be activated and might contribute to the process of progressive heart failure. Since the class I DGKs are characterized by the presence of an EF hand motif (a $\mathrm{Ca}^{2+}$-binding domain) [26], $\mathrm{Ca}^{2+}$ overload, which is one of the key features of a failing heart and which induces mitochondrial disorganization and cardiomyocyte apoptosis [27], might modulate the activity of class I DGKs in failing hearts.

We identified the expression of DGK $\eta$ in the human right atrium but could not detect it in rodent hearts $[14,28]$. Although its functional role is not yet clear, it is noteworthy that the expression of DGK $\eta$ was increased in the failing hearts affected by volume overload. Recently, Yasuda et al. have reported that DGK $\eta$ activates Ras/B-Raf/C-Raf/MEK/ERK signaling pathway by regulating B-Raf-C-Raf heterodimer formation [28], thereby suggesting that increased DGK $\eta$ expression might affect the process of heart failure. Understanding of the role of DGK $\eta$ in human heart failure might be valuable for determining a novel therapeutic target in the future.

Downregulation of DGKE in rat hearts was observed in both myocardial infarction and aortic banding models $[13,14]$. In the present study, expression of DGK $\varepsilon$ was unchanged in the failing human hearts. One possible explanation for this discrepancy is that regulation of DGK isoform expression might be different in different species under different hemodynamic conditions.

In this study, atrial expression of DGK $\zeta$, which belongs to class IV, was significantly decreased in the human hearts affected by volume overload. On the other hand, several contradictory findings have reported in animal models of heart failure. In rat hearts affected by chronic pressure overload, translocation of DGK $\zeta$ from nuclear to cytosolic cell fraction was indicated [13]. DGK $\zeta$ upregulation was reported in the peripheral zone of the necrotic area in infarcted rat hearts [14]. We have previously reported that DGK $\zeta$ mRNA levels in neonatal cardiomyocytes increased in the acute phase, but immediately returned to basal levels after endothelin-1 stimulation [15]. In this study, since the hearts were under continuous strain for a long time due to volume overload, DGK $\zeta$ expression might be decreased in failing human hearts. We have previously reported the importance of DGK $\zeta$ in abrogating the progress of ventricular remodeling. DGK $\zeta$ has been reported to inhibit endothelin-1-induced PKC $\varepsilon$ translocation and hypertrophic responses in neonatal rat cardiomyocytes [15].
Cardiac-specific overexpression of DGK $\zeta$ has been reported to prevent angiotensin II- and phenylepinephrine-induced activation of several PKCs and subsequent cardiac hypertrophy [16]. Our findings may reflect a pathophysiological importance of DGK $\zeta$ in the regulation of cardiac hypertrophy and heart failure in the human heart. On the basis of these facts, we thought that upregulation of DGK $\zeta$ could be a therapeutic target in patients with heart failure.

\section{Conclusions}

In conclusion, this study is the first to provide evidence of differential regulation of human DGK isoforms in failing human heart affected by volume overload, thereby suggesting that individual DGK isoforms may have unique properties, and consequently, distinct functions in the regulation of cardiac hypertrophy and heart failure.

\section{Acknowledgements}

This study was supported, in part, by a grant-in-aid for Scientific Research (No. 21790701, 21590923, and 21590935) from the Ministry of Education, Science, Sports and Culture, Tokyo, Japan, a grant-in-aid from the Global Century Center of Excellence (COE) program of the Japan Society for the Promotion of Science, and grants from The Takeda Science Foundation and Uehara Memorial Foundation, and Japan Heart Foundation Research Grant

\section{Author details}

${ }^{1}$ Department of Cardiology, Pulmonology, and Nephrology, Yamagata University School of Medicine, Yamagata, Japan. ${ }^{2}$ Department of Cardiovascular, Thoracic, and Pediatric Surgery, Yamagata University School of Medicine, Yamagata, Japan. ${ }^{3}$ Department of Cardiology and Hematology, Fukushima Medical University, Fukushima, Japan.

\section{Authors' contributions}

OB and SS carried out the RNA isolation and RT-PCR. TS evaluated the expressions of DGK isoform and compared those expression patterns with rodent. TS and TK compared the medical record regarding clinical and hemodynamic characteristics of patients with heart failure. ST and MS obtained heart samples from patients. YT and IS conceived of the study and participated in its design and coordination. KG participated in the characterization of the DGK isoforms in human. All authors read and approved the final manuscript.

\section{Competing interests}

The authors declare that they have no competing interests.

Received: 1 November 2010 Accepted: 8 May 2011

Published: 8 May 2011

\section{References}

1. Frey N, Katus HA, Olson EN, Hill JA: Hypertrophy of the heart: a new therapeutic target? Circulation 2004, 109:1580-1589.

2. Levy D, Larson MG, Vasan RS, Kannel WB, Ho KK: The progression from hypertension to congestive heart failure. JAMA 1996, 275:1557-1562.

3. Kannel WB, Cobb J: Left ventricular hypertrophy and mortality-results from the Framingham Study. Cardiology 1992, 81:291-298.

4. Dorn GW, Force T: Protein kinase cascades in the regulation of cardiac hypertrophy. J Clin Invest 2005, 115:527-537.

5. Hunter JJ, Chien KR: Signaling pathways for cardiac hypertrophy and failure. N Engl J Med 1999, 341:1276-1283.

6. Kilts JD, Grocott HP, Kwatra MM: G alpha(q)-coupled receptors in human atrium function through protein kinase $\mathrm{C}$ epsilon and delta. J Mol Cell Cardiol 2005, 38:267-276. 
7. Mende U, Kagen A, Cohen A, Aramburu J, Schoen FJ, Neer EJ: Transient cardiac expression of constitutively active Galphaq leads to hypertrophy and dilated cardiomyopathy by calcineurin-dependent and independent pathways. Proc Natl Acad Sci USA 1998, 95:13893-13898.

8. Takeishi $Y$, Jalili T, Ball NA, Walsh RA: Responses of cardiac protein kinase $\mathrm{C}$ isoforms to distinct pathological stimuli are differentially regulated. Circ Res 1999, 85:264-271.

9. Goto K, Kondo H: A 104-kDa diacylglycerol kinase containing ankyrin-like repeats localizes in the cell nucleus. Proc Natl Acad Sci USA 1996, 93:11196-11201

10. Kanoh H, Yamada K, Sakane F: Diacylglycerol kinases: emerging downstream regulators in cell signaling systems. J Biochem 2002, 131:629-633.

11. Sakane F, Imai S, Kai M, Yasuda S, Kanoh H: Diacylglycerol kinases: why so many of them? Biochim Biophys Acta 2007, 1771:793-806.

12. Takeishi Y, Goto K, Kubota I: Role of diacylglycerol kinase in cellular regulatory processes: A new regulator for cardiomyocyte hypertrophy. Pharmacol Ther 2007, 115:352-359.

13. Yahagi H, Takeda M, Asaumi Y, Okumura K, Takahashi R, Takahashi J, Ohta J, Tada H, Minatoya Y, Sakuma M, Watanabe J, Goto K, Shirato K, Kagaya Y: Differential regulation of diacylglycerol kinase isozymes in cardiac hypertrophy. Biochem Biophys Res Commun 2005, 332:101-108.

14. Takeda M, Kagaya Y, Takahashi J, Sugie T, Ohta J, Watanabe J, Shirato K, Kondo H, Goto K: Gene expression and in situ localization of diacylglycerol kinase isozymes in normal and infarcted rat hearts: effects of captopril treatment. Circ Res 2001, 89:265-272.

15. Takahashi H, Takeishi $Y$, Seidler T, Arimoto T, Akiyama H, Hozumi Y, Koyama Y, Shishido T, Tsunoda Y, Niizeki T, Nozaki N, Abe J, Hasenfuss G, Goto K, Kubota I: Adenovirus-mediated overexpression of diacylglycerol kinase-zeta inhibits endothelin-1-induced cardiomyocyte hypertrophy. Circulation 2005, 111:1510-1516.

16. Arimoto T, Takeishi $Y$, Takahashi H, Shishido T, Niizeki T, Koyama Y, Shiga R, Nozaki N, Nakajima O, Nishimaru K, Abe J, Endoh M, Walsh RA, Goto K, Kubota I: Cardiac-specific overexpression of diacylglycerol kinase zeta prevents Gq protein-coupled receptor agonist-induced cardiac hypertrophy in transgenic mice. Circulation 2006, 113:60-66.

17. Shishido T, Nozaki N, Takahashi H, Arimoto T, Niizeki T, Koyama Y, Abe J, Takeishi Y, Kubota I: Central role of endogenous Toll-like receptor-2 activation in regulating inflammation, reactive oxygen species production, and subsequent neointimal formation after vascular injury. Biochem Biophys Res Commun 2006, 345:1446-1453.

18. Nozaki N, Shishido T, Takeishi Y, Kubota I: Modulation of doxorubicininduced cardiac dysfunction in toll-like receptor-2-knockout mice. Circulation 2004, 110:2869-2874

19. Imai S, Kai M, Yasuda S, Kanoh H, Sakane F: Identification and characterization of a novel human type II diacylglycerol kinase, DGK kappa. J Biol Chem 2005, 280:39870-39881.

20. Miyamoto T, Takeishi Y, Tazawa S, Inoue M, Aoyama T, Takahashi H, Arimoto $T$, Shishido T, Tomoike $H$, Kubota I: Fatty acid metabolism assessed by 125I-iodophenyl 9-methylpentadecanoic acid (9MPA) and expression of fatty acid utilization enzymes in volume-overloaded hearts. Eur J Clin Invest 2004, 34:176-181.

21. Yan C, Ding B, Shishido T, Woo CH, Itoh S, Jeon Kl, Liu W, Xu H, McClain C, Molina CA, Blaxall BC, Abe J: Activation of extracellular signal-regulated kinase 5 reduces cardiac apoptosis and dysfunction via inhibition of a phosphodiesterase 3A/inducible cAMP early repressor feedback loop. Circ Res 2007, 100:510-519.

22. Miyamoto T, Takeishi Y, Takahashi H, Shishido T, Arimoto T, Tomoike H, Kubota l: Activation of distinct signal transduction pathways in hypertrophied hearts by pressure and volume overload. Basic Res Cardiol 2004, 99:328-337.

23. Alexopoulos D, Lazzam C, Borrico S, Fiedler L, Ambrose JA: Isolated chronic mitral regurgitation with preserved systolic left ventricular function and severe pulmonary hypertension. J Am Coll Cardiol 1989, 14:319-322.

24. Ding B, Abe J, Wei H, Huang Q, Walsh RA, Molina CA, Zhao A, Sadoshima J, Blaxall BC, Berk BC, Yan C: Functional role of phosphodiesterase 3 in cardiomyocyte apoptosis: implication in heart failure. Circulation 2005, 111:2469-2476.

25. Takeishi Y, Huang Q, Abe J, Che W, Lee JD, Kawakatsu H, Hoit BD, Berk BC, Walsh RA: Activation of mitogen-activated protein kinases and $\mathrm{p} 90$ ribosomal $\mathrm{S} 6$ kinase in failing human hearts with dilated cardiomyopathy. Cardiovasc Res 2002, 53:131-137.

26. Yamada K, Sakane F, Matsushima N, Kanoh H: EF-hand motifs of alpha, beta and gamma isoforms of diacylglycerol kinase bind calcium with different affinities and conformational changes. Biochem J 1997 321:59-64.

27. Palaniyandi SS, Sun L, Ferreira JC, Mochly-Rosen D: Protein kinase C in heart failure: a therapeutic target? Cardiovasc Res 2009, 82:229-239.

28. Yasuda S, Kai M, Imai S, Takeishi K, Taketomi A, Toyota M, Kanoh H, Sakane F: Diacylglycerol kinase eta augments C-Raf activity and B-Raf/CRaf heterodimerization. J Biol Chem 2009, 284:29559-29570.

doi:10.1186/1749-8090-6-65

Cite this article as: Bilim et al:: Differential regulation of diacylglycerol kinase isoform in human failing hearts. Journal of Cardiothoracic Surgery 2011 6:65.

\section{Submit your next manuscript to BioMed Central and take full advantage of:}

- Convenient online submission

- Thorough peer review

- No space constraints or color figure charges

- Immediate publication on acceptance

- Inclusion in PubMed, CAS, Scopus and Google Scholar

- Research which is freely available for redistribution

Submit your manuscript at www.biomedcentral.com/submit
C) Biomed Central 